\title{
Beyond dimensional analysis: Higgs and new Higgs inflations do not violate unitarity
}

\author{
Albert Escrivà $^{1,2, *}$ and Cristiano Germani ${ }^{1, \dagger}$ \\ ${ }^{1}$ Institut de Ciències del Cosmos, Universitat de Barcelona, Martí i Franquès 1, 08028 Barcelona, Spain \\ ${ }^{2}$ Departament de Física Quàntica i Astrofísica, Facultat de Física, Universitat de Barcelona, \\ Martí i Franquès 1, 08028 Barcelona, Spain
}

(Received 25 December 2016; published 20 June 2017)

\begin{abstract}
Naive dimensional analysis seems to suggest possible unitarity violations in the framework of the Higgs and new Higgs inflationary scenarios. These violations seem to happen around the value in which the potential energy, per a given Higgs boson's vacuum expectation value, crosses the perturbative cutoff scale calculated around the electroweak vacuum. Conversely to these expectations, we show that, by using an exact analysis of the background dependent cutoff scale, and by including the contribution of the phasespace volume in the perturbative scattering amplitudes of scalars, no violation of (perturbative) unitarity might ever happen during the whole Universe evolution.
\end{abstract}

DOI: 10.1103/PhysRevD.95.123526

\section{THE NAIVE STORY}

Because inflation is supposed to generate a homogeneous and isotropic Universe (at the background level), a natural inflaton candidate is a spin 0 particle. The standard model (SM) of particle physics contains already a scalar field: the Higgs boson. Therefore one may be tempted to consider the very economical scenario in which the Higgs boson is also the inflaton (this is a very old story, see for example [1]). However, as is well known, the Higgs boson has too steep of a potential to generate a successful inflation. Assuming no extra degrees of freedom in nature, rather than the ones of the standard model of particle physics and gravity, at least up to the inflationary scale, leads to only two possibilities. The first one is that the actual Higgs potential becomes shallower after certain scales (Higgs inflation) [1] (for this scenario with current precision data see [2]), and the other is that the gravitational friction acting on the Higgs boson is stronger than that already provided by standard general relativity (new Higgs inflation) [3]. After this is achieved at tree level, loop analysis can be done such to consider the contribution of the running coupling constants to inflation (see [2] for Higgs inflation and [4] for new Higgs inflation).

However, the use of these scenarios has already been criticized on the basis of a putative perturbative unitarity violation (for the latest rebuttal on this see [5]). The story goes along these lines ${ }^{1}$ : Although the Higgs inflationary scenarios only make use of the SM degrees of freedom and gravity, they are nonrenormalizable effective field theories

\footnotetext{
*albert.escriva@fqa.ub.edu

†ermani@icc.ub.edu

${ }^{1}$ Strictly speaking this has been extensively analyzed only in the Higgs inflation of [1]. However, the same qualitative analysis can be done in the new Higgs inflation [3].
}

(EFTs). The Higgs boson and/or the graviton are no canonical degrees of freedom in these EFTs. Therefore, in order to figure out the perturbative unitarity violation scale, one has to diagonalize the graviton-Higgs system and, then, check the scales $\left(V_{m}\right)$ suppressing the nonrenormalizable operators constructed with $m$ powers of the diagonalized field fluctuations. This procedure is however background dependent and so are the vertices $V_{m}$.

The diagonalization of the Higgs-gravity system does not have an analytical form. For this reason, the usual approach has been to consider the perturbative cutoffs $\left(\Lambda_{m}\right)$ constructed on the vertices $V_{m}$ only around the electroweak $(\phi=v)$ and large Higgs vacuum expectation value (vev) $\left(\phi=\phi_{\ell}\right)$ scales.

Within the above approximation, one finds that the naive cutoff in dimensional analysis, i.e. for $\Lambda_{m} \sim V_{m}$, is obtained in the $m \rightarrow \infty$ limit. In particular, since cutoffs are also background dependent, one readily finds $\Lambda_{v}<\Lambda_{\ell}$.

As the potential energy of the Higgs boson becomes larger than $\Lambda_{v}$ for $\phi \ll \phi_{\ell}$, extrapolating the behavior of the cutoff in the transition region $v<\phi<\phi_{\ell}$, standard lore was to advocate for a perturbative unitarity violation there.

The "reason" is the following: The fact that the background energy is larger than the perturbative cutoff around the background chosen does not mean by itself that unitarity is violated. However, quite conservatively, one might consider that the potential energy of the background represents a reservoir energy available for random scatterings between scalar fluctuations. In this case, there can be a nonvanishing probability that a scattering with momenta of order of the background energy happens, thus violating unitarity. Obviously, this conclusion may be very superficial as it does not really follow from a thorough examination of high energy scatterings in this system (see for example [6,7]).

In any case, even blindly using the argument that the potential energy density sets the maximal momenta for 
random scatterings, we show that the conclusion of unitarity violation of these systems is premature. The main reason is that the exact background cutoffs, calculated by considering the unitarity bounds of tree-level scattering amplitudes, instead of the naive dimensional analysis in previous literature, show unequivocally that the potential energy is always way below the perturbative unitarity violating scale. Specifically, for large $m$ 's, $\Lambda_{m} \gg V_{m}$ instead of the dimensional analysis $\Lambda_{m} \sim V_{m}$. We will prove this by considering $2 \rightarrow n$ scatterings.

Although the question of whether the potential of Higgs inflationary scenarios is fine-tuned or not still remains, our results show that Higgs and new Higgs inflationary scenarios are robust EFTs during the whole Universe evolution.

\section{HIGGS INFLATION}

In the Higgs inflationary scenario the Higgs boson's $(\phi)$ action, at large field values (in the unitary gauge, neglecting SM couplings and the Higgs mass), is

$$
S_{H}=\frac{1}{2} \int d^{4} x \sqrt{-\tilde{g}}\left[M_{p}^{2}\left(1+\frac{\xi \phi^{2}}{M_{p}^{2}}\right) \tilde{R}-\tilde{\partial}_{\alpha} \phi \tilde{\partial}^{\alpha} \phi-\frac{\lambda}{2} \phi^{4}\right],
$$

where $M_{p}=2.435 \times 10^{18} \mathrm{GeV}$ is the Planck mass, $(\tilde{)})$ indicates content evaluated with the metric $\tilde{g}, \lambda \simeq 0.13$ is the tree-level Higgs quartic coupling and finally $\xi \gg 1$ is a constant defining a new mass scale $M=M_{p} \xi^{-1 / 2}$. Note that the conformal term multiplying the Ricci scalar $R$ is not unique as we could have chosen any function of $\phi$. We will however follow [1] for our analysis.

Although it might seem that in the above action the Higgs boson is nonminimally coupled to gravity, it is not. The reason is that in the canonical system, the so-called Einstein frame, the field $\phi$ is minimally coupled.

By making the conformal rescaling $g_{\alpha \beta}=\Omega^{2} \tilde{g}_{\alpha \beta}$, with $\Omega^{2}=\left(1+\frac{\xi \phi^{2}}{M_{p}^{2}}\right)$, we will end up with the canonical action

$$
S_{H}=\frac{1}{2} \int d^{4} x \sqrt{-g}\left[M_{p}^{2} R-\partial_{\alpha} \chi \partial^{\alpha} \chi-2 U(\chi)\right],
$$

where the relation between the new canonical variable $\chi$, the Higgs boson and the new potential $U$ is

$$
\begin{aligned}
\frac{d \chi}{d \phi} & =\sqrt{\frac{\Omega^{2}+6 \xi^{2} \phi^{2} / M_{p}^{2}}{\Omega^{4}},} \\
U(\chi) & =\frac{\lambda}{4} \frac{\phi(\chi)^{4}}{\left(1+\frac{\xi \phi(\chi)^{2}}{M_{p}^{2}}\right)^{2}} .
\end{aligned}
$$

After this field redefinition, we see that the nonrenormalizability of the action is codified by a nonrenormalizable potential (and of course by gravity). It is easy to see that for small values of the Higgs vacuum expectation value $\phi \ll M / \sqrt{\xi}$, the potential is well approximated by the standard electroweak potential and the Higgs boson is approximately canonical, while for large vev's $\phi \gg M$ the potential becomes exponentially flat so as to accommodate inflation.

From the perspective of perturbative analysis as seen from the electroweak vacuum, i.e. expanding the Higgs boson potential around the $\chi=0 \mathrm{vev}$, perturbative unitarity is violated at the energy $M / \sqrt{\xi}$. Therefore, one may ask the question of whether perturbative unitarity is violated somewhere before reaching the energy scales of interest for inflation. A second question would then be whether or not the modified Higgs potential is fine-tuned at the one or more loop level. Here, we will only focus on the question of whether perturbative unitarity is violated and leave the question of possible fine-tunings for future work.

In principle, the fact that the Higgs vev is large, implying a "large" energy density, does not necessarily imply a violation of unitarity, as we have already discussed. However, one might argue that the energy of the background should always be below the perturbative cutoff energy scale. We will not discuss here whether this assumption is correct or not, what we will show is that, even accepting this conservative prejudice, neither the Higgs nor the new Higgs inflationary scenarios violate perturbative unitarity during the whole Universe evolution. The reason is that in these scenarios, the unitarity violating scale is a background dependent quantity as we shall now discuss.

The procedure to obtain the background dependent cutoff is to expand the potential in some background $\chi=\chi_{0}+\delta \chi$,

$$
U\left(\chi+\chi_{0}\right)=U\left(\chi_{0}\right)+\left.\sum_{m=1}^{\infty} \frac{1}{m !} \frac{d^{m} U(\chi)}{d \chi^{m}}\right|_{\chi=\chi_{0}}(\delta \chi)^{m},
$$

and then calculate the cross sections $\sigma$ of $\delta \chi$ particles due to any nonrenormalizable vertices. In particular, without lost of generality, we will focus on the $2 \rightarrow n$ scatterings with $n>2$. The unitarity bound is then

$$
\sigma[2 \rightarrow n] \leq \frac{4 \pi}{s},
$$

where $\sigma$ is the cross section of the process.

The structure of these scattering amplitudes are very reminiscent of the multiple-particle scattering amplitudes of would-be Goldstone bosons in the electroweak theory, after symmetry breaking. There, dimensional analysis would suggest that the $2 \rightarrow n$ scattering, at large $n$, would saturate the unitarity bound at the electroweak breaking scale $v$. However, kinematic conditions suggest at least a linear growing with the number of particles. This puzzle was resolved by [8] by noticing that dimensional analysis is 
grossly wrong for large $n$ 's due to the neglected phasespace volume contribution to the scattering amplitudes. Precisely the same fact that helped unitarity in the large $n$ scatterings in electroweak theory will solve the unitarity issues in Higgs inflationary models.

Here we will only focus on contact diagrams. Note that, in the $2 \rightarrow n$ scattering amplitudes, cascades of tree-level amplitudes with lower number of particles also contribute. There, the sum of those diagrams could violate unitarity. However, if every single vertex does not, by doing a careful (Borel) summation, the final scattering amplitude should not violate unitarity either. Nevertheless, this is an old issue that still afflicts even renormalizable theories, e.g. the standard electroweak $\phi^{4}$ theory. By assuming that this computational matter is solved in quantum field theory, we will not further investigate it here. Indeed, this issue is far beyond the scope of the present paper, which aims to show that contact diagrams do not violate unitarity, as conversely claimed in the previous literature. In addition, as we are not aiming to find the precise value of the scattering amplitude of $2 \rightarrow n$ scatterings, but rather just check for unitarity, we will not consider cascading diagrams.

Taking into account the symmetry factors due to the fact that we are considering a scattering of $m=n+2$ particles, the vertex involving $m$ particles is

$$
V_{m}\left(\chi_{0}\right)=\left(\frac{d^{m} U\left(\chi_{0}\right)}{d \chi^{m}}\right)^{\frac{1}{4-m}}
$$

The perturbative unitarity bound is then saturated at the energy (ignoring angular dependence)

$$
\Lambda_{m}\left(\chi_{0}\right)=\mathcal{O}(1)\left|V_{m}\left(\chi_{0}\right)\right| F_{m}^{\frac{1}{2(m-2)}},
$$

where the factor

$$
F_{m}=2^{4 m-10} \pi^{2 m-6}(m-3) !(m-4) !
$$

comes form the phase-space integration [8]. ${ }^{2}$

Interestingly, $F_{m}^{\frac{1}{2(m-2)}} \rightarrow \frac{4 \pi m}{e}$ for large $m$ 's, making the perturbative cutoff linearly growing with the number of particles involved in the scattering. This fact was completely overlooked in the literature, for Higgs and new Higgs inflationary scenarios. That led to wrong claims about the perturbative unitarity violation in Higgs and new Higgs inflationary scenarios.

Forgetting the $\mathcal{O}(1)$ contribution to the perturbative cutoff, ${ }^{3}$ we can now check whether $f_{m}\left(\chi_{0}\right) \equiv \frac{U\left(\chi_{0}\right)^{1 / 4}}{\Lambda_{m}\left(\chi_{0}\right)}$ ever exceeds one signaling a possible unitarity violation in the

\footnotetext{
${ }^{2}$ Note that, conversely to [8] we are rewriting the scattering amplitude in a function of $m$ and not $n$. In addition, since we are considering equivalent particles, the $\delta$ of [8] is 2 .

${ }^{3}$ This is very conservative as the $\mathcal{O}(1)$ will help unitarity.
}

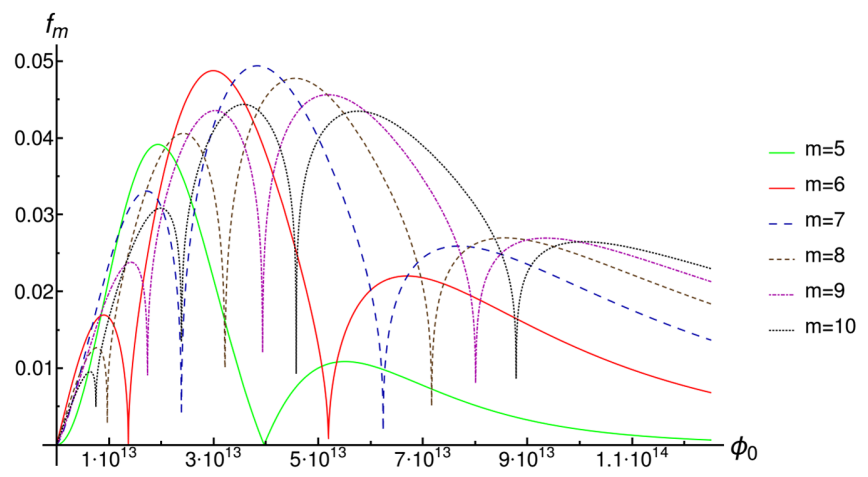

FIG. 1. Numerical computation of the $f_{m}$ 's for Higgs inflation. The parameter $\xi \simeq 1.8 \times 10^{4}$ is obtained from [9] by using $n_{s} \simeq$ 0.9655 [10]. The picks corresponds to 0 s of the $f_{m}$ 's.

background chosen. As can be seen from Fig. 1, there is a maximum of $f\left(\chi_{0}\right)$ close to where we would expect it, i.e. around the vev $\phi_{c} \sim M_{p} / \xi$ (the exact value depends on the vertex chosen). In the same figure we can see that the unitarity is never violated, at least up to $m=10$. What is even more interesting is that for $m \geq 7$ the maximum values decrease and move to the right. Then the $m$ curve recrosses the $(m-1)$ 's at values of $\phi$ increasing with $m$. However, this happens in the unitarity region. Unfortunately, due to the complexity of the function $\chi(\phi), \Lambda_{m}$ 's with $m>10$ are exponentially expensive to calculate.

Because of the shifting of the recross point towards large $\phi$ values, our intuition from the $m \leq 10$ curves is that there is always an $m_{*}(h)$ such that $\frac{f_{m_{*}}}{f_{m>m_{*}}}>1$, where $\frac{d m_{*}}{d h}>0$. If this is true, then $\operatorname{Max}\left[f_{m}\left(\chi_{0}\right)\right] \sim f_{7}\left(\chi\left(\phi_{c}\right)\right)<1$ and thus no unitarity violation may happen.

To back up our intuition we will rely on some approximation that can be done in the innocuous region ${ }^{4}$ $M_{p} / \xi \ll \phi \ll M_{p} / \sqrt{\xi}$. There, for large $m$ 's, $V_{m} \approx$ $\frac{\phi^{2} \xi}{M_{p}}\left[\frac{\xi^{6} \phi^{6}}{\lambda M_{p}^{6}}\right]^{1 /(m-4)}$ [7]. ${ }^{5}$ It is then easy to check that, in this approximation and for the value of the parameter chosen in Fig. $1, \frac{f_{m_{*}}}{f_{m>m_{*}}}>1$, with $m_{*}=34$, which backs up our expectations.

Concluding, we have shown that, within the Higgs inflationary model, no unitarity is violated in scalar scatterings. Note that the same conclusion was guessed by $[7,11]$. However, as we have seen, the maximal peak of $f_{m}$ happens around $\phi_{c} \sim M_{p} / \sqrt{\xi}$. Precisely here, the approximated analysis of [7,11] is invalid. In addition, our numerical results show that, if we had used $V_{m}$ instead of $\Lambda_{m}$ as in $[7,11]$, we would have probably obtained a violation of unitarity.

\footnotetext{
${ }^{4}$ In other words, in the region in which $f_{m}<1$ for all $m$ 's.

${ }^{5}$ In passing we noted that the computation of [11] here is wrong.
} 


\section{NEW HIGGS INFLATION}

In the new Higgs inflation of [3] the action is

$$
\begin{aligned}
S_{n H}= & \frac{1}{2} \int d^{4} x \sqrt{-\tilde{g}} \\
& \times\left[M_{p}^{2} \tilde{R}-\left(\tilde{g}^{\alpha \beta}-\frac{\tilde{G}^{\alpha \beta}}{M^{2}}\right) \tilde{\partial}_{\alpha} \phi \tilde{\partial}_{\beta} \phi-2 V(\phi)\right],
\end{aligned}
$$

with $V(\phi)=\frac{\lambda}{4} \phi^{4}$. However, in this form, the previous analysis is not directly applicable.

By looking at the Hubble equations (see for example [3]) on a homogeneous and isotropic background, one can easily find the bound $\dot{\phi}^{2}<\frac{2}{3} M^{2} M_{p}^{2}$. In fact, on average, $\left\langle\dot{\phi}^{2}\right\rangle \ll \frac{2}{3} M^{2} M_{p}^{2}$ [12]. As discussed before, we are interested in the case in which some canonical scalar quanta could spontaneously scatter with up to the background energy. Using the same notation as used previously, we are therefore interested in the case in which $\partial_{\alpha} \delta \chi \partial^{\alpha} \delta \chi \leq U\left(\chi_{0}\right)$. As we shall soon see, the relation between $\chi$ and $\phi$ is $(\partial \chi)^{2}=\left(1+\frac{V(\phi)}{M^{2} M_{p}^{2}}\right)(\partial \phi)^{2}$, where $V(\phi)=\frac{1}{4} \lambda \phi^{4}$ and $U(\chi) \equiv V(\phi(\chi))$. Then, we immediately see that, in our region of interest, $(\partial \delta \phi)^{2}<M^{2} M_{p}^{2}$, thus $\frac{\partial_{\alpha} \phi \partial_{\beta} \phi}{M^{2} M_{p}^{2}}<1$. Although this might already be enough to solve unitarity issues in the new Higgs inflation [13], we will once again discuss the validity of this EFT in terms of scattering amplitudes.

In this case we can use a disformal transformation of the metric [14] $g_{\alpha \beta}=\tilde{g}_{\alpha \beta}+\frac{\partial_{\alpha} \phi \partial_{\beta} \phi}{M^{2} M_{p}^{2}}$, to obtain

$$
\begin{aligned}
S_{n H}= & \frac{1}{2} \int d^{4} x \sqrt{-g} \\
& \times\left[M_{p}^{2} R-\left(1+\frac{V(\phi)}{M^{2} M_{p}^{2}}\right) \partial_{\alpha} \phi \partial_{\beta} \phi-2 V(\phi)\right. \\
& + \text { higher powers of derivatives }],
\end{aligned}
$$

where the operators with higher powers of derivatives will be suppressed by higher powers of $M^{2} M_{p}^{2}$. Since we are interested in the leading contribution to the $m$-particle scatterings and we do not aim for precision physics, we will neglect those.

At this point, the discussion is immediately connected to the previous one with

$$
\begin{aligned}
\frac{d \chi}{d \phi} & =\sqrt{1+\frac{V(\phi)}{M^{2} M_{p}^{2}},} \\
U(\chi) & =V(\phi(\chi)) .
\end{aligned}
$$

Once the disformal transformation is performed, the good thing is that in the new Higgs inflation the computation of the vertices is not expensive, thus we can numerically plot

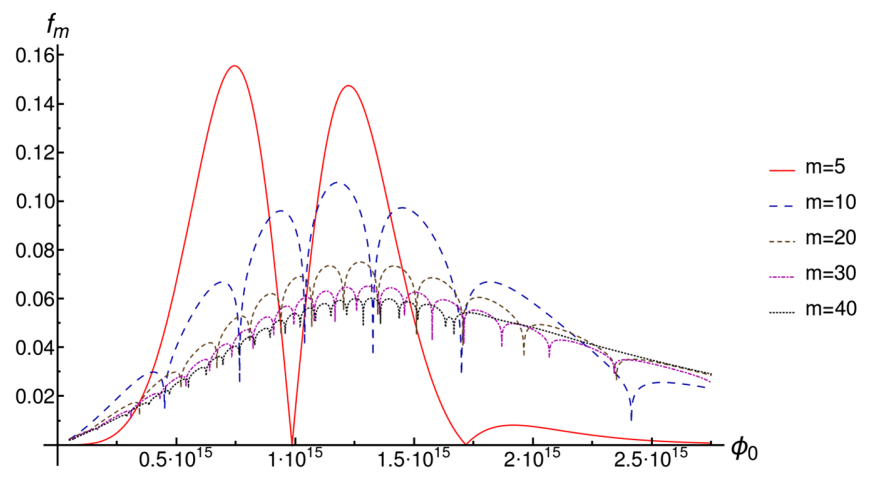

FIG. 2. Numerical computation of the $f_{m}$ 's for new Higgs inflation. The parameter $M \simeq 7.2 \times 10^{10} \mathrm{GeV}$ is obtained from [15] by using $n_{s} \simeq 0.9655$ [10]. As before, the picks corresponds to $0 \mathrm{~s}$ of the $f_{m}$ 's.

the $f_{m}$ 's even for large $m$ values. In this case, as can be seen from Fig. 2, $f_{m_{1}}>f_{m_{2}}$ for any $m_{1}>m_{2}$. Then, since unitarity is not violated for $f_{5}$, it never is.

\section{CONCLUSION}

It has been a several years long debate, starting with [16] and continuing through [5], as to whether or not the Higgs inflation of [17] violates tree-level unitarity. Lately, by using some nonperturbative techniques, Calmet and Casadio [18] studied loop corrections to the Higgs inflation and found indications that unitarity might not be violated, order by order. In this paper we show that this indeed happens, i.e. Higgs inflation has not violated perturbative unitarity during the whole history of the Universe.

The missing ingredients of previous analysis were basically two: The first one was simply computational. All analysis done before the current paper was based on extrapolations of the background dependent cutoff behavior on the region of the putative unitarity violation. The exact numerical calculation deviates from this.

The second and crucial missing piece was the actual calculation of the unitarity bound. All previous analysis were only performed by the use of dimensional analysis. In other words, they were only based on checking whether the potential energy of the background would become larger than the suppression scales appearing in the expanded Higgs inflationary scenario, around the background chosen. There, typically, the violation of unitarity would appear in correspondence to the highest dimensional operator suppressed by a scale $V_{m}$, i.e. in a scattering of a large number of $(m)$ particles.

However, in the latter case, the phase-space volume linearly grows with $m$, making the true cutoff $\Lambda_{m}$ also grow with $m$, i.e. $\Lambda_{m} \sim m V_{m}$. Contrary to the standard lore, we show that larger and larger dimensional operators correspond to more and more unitary scatterings. This proves the Higgs inflation to be unitary during the whole Universe evolution. 
We then analyze the new Higgs inflationary scenario of [3], where the Higgs boson is derivatively coupled to curvature. There, we show that, at least during the evolution of the Universe, a disformal transformation of the metric can be done so to find an approximate Einstein frame. In this case we were able to apply the same analysis performed in the Higgs inflationary case finding no perturbative unitarity violation for the entire history of the Universe.

A final comment is due here. In unitary gauge, the Higgs boson is a real scalar field $\phi$ coupled to gauge bosons. In Higgs inflation, at large field values (see [7]) the mass scale of non-Abelian vectors is $M_{V} \simeq g M_{p} / \sqrt{\xi}$, where $g \ll 1$ is the non-Abelian (strong) coupling. In this sector unitarity is then violated at a scale $\Lambda_{V} \sim M_{V} / g$. Because $\lambda \ll 1$, the cutoff scale in the vector scatterings is parametrically smaller than in the scalar sector, but still far larger than the scalar potential during inflation. Thus, no unitarity violation is expected there.

In new Higgs inflation the situation is much better as, for large field values, the gauge bosons mass scale is parametrically larger than the scalar cutoff, see [15].

\section{ACKNOWLEDGMENTS}

C. G. is supported by the Ramon y Cajal program and partially supported by the Unidad de Excelencia María de Maeztu Grants No. MDM-2014-0369 and No. FPA201346570-C2-2-P. C. G. would like to thank the Yukawa Institute for Theoretical Physics for its hospitality and Alex Kehagias for the comments. A. E. thanks Federico Mescia for the useful discussions.
[1] D. S. Salopek, J. R. Bond, and J. M. Bardeen, Phys. Rev. D 40, 1753 (1989).

[2] F. Bezrukov, J. Rubio, and M. Shaposhnikov, Phys. Rev. D 92, 083512 (2015); F. Bezrukov and M. Shaposhnikov, J. High Energy Phys. 07 (2009) 089.

[3] C. Germani and A. Kehagias, Phys. Rev. Lett. 105, 011302 (2010).

[4] S. Di Vita and C. Germani, Phys. Rev. D 93, 045005 (2016).

[5] A. Kehagias, A. Moradinezhad Dizgah, and A. Riotto, Phys. Rev. D 89, 043527 (2014).

[6] Z.-Z. Xianyu, J. Ren, and H.-J. He, Phys. Rev. D 88, 096013 (2013); J. Ren, Z.-Z. Xianyu, and H.-J. He, J. Cosmol. Astropart. Phys. 06 (2014) 032.

[7] F. Bezrukov, A. Magnin, M. Shaposhnikov, and S. Sibiryakov, J. High Energy Phys. 01 (2011) 016.

[8] D. A. Dicus and H.-J. He, Phys. Rev. D 71, 093009 (2005); Phys. Rev. Lett. 94, 221802 (2005).

[9] F. Bezrukov, D. Gorbunov, and M. Shaposhnikov, J. Cosmol. Astropart. Phys. 06 (2009) 029.
[10] P. A. R. Ade et al. (Planck Collaboration), Astron. Astrophys. 594, A13 (2016).

[11] S. Ferrara, R. Kallosh, A. Linde, A. Marrani, and A. Van Proeyen, Phys. Rev. D 83, 025008 (2011).

[12] C. Germani, N. Kudryashova, and Y. Watanabe, J. Cosmol. Astropart. Phys. 08 (2016) 015.

[13] C. Germani and Y. Watanabe, J. Cosmol. Astropart. Phys. 07 (2011) 031; 07 (2011) A01.

[14] C. Germani, L. Martucci, and P. Moyassari, Phys. Rev. D 85, 103501 (2012).

[15] C. Germani, Y. Watanabe, and N. Wintergerst, J. Cosmol. Astropart. Phys. 12 (2014) 009.

[16] J. L. F. Barbon and J. R. Espinosa, Phys. Rev. D 79, 081302 (2009); C. P. Burgess, H. M. Lee, and M. Trott, J. High Energy Phys. 07 (2010) 007; M. P. Hertzberg, J. High Energy Phys. 11 (2010) 023.

[17] F. L. Bezrukov and M. Shaposhnikov, Phys. Lett. B 659, 703 (2008).

[18] X. Calmet and R. Casadio, Phys. Lett. B 734, 17 (2014). 